\title{
Atomistic Modelling of Segregation and Precipitation in Fe-Cr Alloys under Irradiation
}

\author{
Frédéric SOISSON ${ }^{1}$, Chu Chun FU ${ }^{1}$, Thomas JOURDAN ${ }^{1}$, Maylise NASTAR ${ }^{1}$, \\ Oriane SENNINGER ${ }^{1}$, Enrique MARTINEZ ${ }^{2}$, Yves BRECHET ${ }^{3}$ \\ ${ }^{1}$ CEA-DEN-DMN, Service de Recherches de Métallurgie Physique, SRMP (Saclay, France) \\ ${ }^{2}$ Los Alamos National Laboratory, Materials Science in Radiation Dynamics Group (Los Alamos, USA) \\ SIMAP, INP Grenoble, CNRS UJF (Saint Martin d'Hères, France)
}

Iron-chromium alloys are the basis for ferritic and ferritic-martensitic steels that will be used in future fission (generation IV) and fusion nuclear reactors. With $\mathrm{Cr}$ content between typically 8 to 12\% [1], or even $14 \%$ in the matrix of some oxide dispersion-strengthened steels [2], one can expect the precipitation of a Cr-rich a' phase that can be strongly accelerated under irradiation, due to point defect supersaturation. This precipitation can cause hardening and embrittlement. Radiation-induced segregation (RIS) is another important technological problem. It can lead to a $\mathrm{Cr}$ depletion at grain boundaries and therefore to a loss of corrosion resistance and again, to embrittlement. RIS in austenitic steels is well known and presents almost systematic trends: depletions of $\mathrm{Cr}$ and enrichments of $\mathrm{Ni}$ at grain boundaries. In ferritic steels, the experimental situation is far from being so clear: depletions and enrichments of $\mathrm{Cr}$ have been observed, without clear correlation with the irradiation conditions and the materials properties [3].

Segregation and precipitation occur by formation, migration and elimination of point defects (vacancies and self-interstitials). We present a model that includes these mechanisms, and combines $\mathrm{ab}$ initio and atomistic kinetic Monte Carlo (AKMC) simulations. Migration barriers and jump frequencies are computed by using effective pair interactions with concentration and temperature dependences. Thanks to these dependences, the simulations are able to well reproduce the thermodynamic [4] and diffusion [5] properties of dilute and concentrated alloys, including the effects of the magnetic configurations and magnetic transitions, which are especially important in $\mathrm{Fe}-\mathrm{Cr}$ alloys. They also give kinetics pf precipitation during isothermal annealing in good agreement with experimental studies [5].

Simulations of $\alpha^{\prime}$ precipitation under irradiation, predict that the kinetics can be considerably accelerated. For example, irradiations of alloys with 15 to $18 \% \mathrm{Cr}$ at $290^{\circ} \mathrm{C}$ and a dose rate of $3.4 \times 10^{-7}$ dpa.s ${ }^{-1}$ produce a point defect supersaturations and therefore, an acceleration, of approximately 6-7 orders of magnitudes. To get a more precise estimation of the evolution of sink densities and point defect concentrations, cluster dynamics [6] have been used: the physical time scale of the Monte Carlo simulations is rescaled accordingly and one gets precipitation kinetics in good agreement with the few available experimental results $[7,8]$. The possible effect of ballistic mixing occurring in displacement cascades and the role of carbon, which is known to strongly interact with the vacancies, will be discussed.

RIS results from the elimination of excess vacancies $(V)$ and self-interstitials (I) on sinks such as dislocations, grain boundaries, or free surfaces. Permanent fluxes of point defects towards the sinks are then sustained, i.e. permanent fluxes of chemical species, leading to a modification of the local composition near the sinks. RIS can be analyzed properly in the framework of the thermodynamics of irreversible processes, that gives the flux of a specie $\alpha$ (atom or defect) as a function of the gradients of chemical potentials $\mu_{\beta}: J_{\alpha}=-\sum L_{\alpha \beta} \nabla \mu_{\beta}$. The tendencies to solute enrichment or depletion near the sinks are controlled by the Onsager coefficients, $L_{\alpha \beta}$. In Fe-Cr alloys, one must observe a depletion of $\mathrm{Cr}$ near the sinks if $\mathrm{L}_{\mathrm{CrV}} / \mathrm{L}_{\mathrm{FeV}}>\mathrm{L}_{\mathrm{Crl}} / \mathrm{L}_{\mathrm{Fel}}$, an enrichment of $\mathrm{Cr}$ in the opposite case. Onsager coefficients can be directly estimated in Monte Carlo simulations (Fig. 1a). Simulations have been also performed to model directly the evolution of point defects and solute distributions near a grain boundary (Fig. 1b). As expected from the analysis of the Onsager coefficients, at $650 \mathrm{~K}$ the interstitial contribution dominates and one observes an enrichment of $\mathrm{Cr}$. On the contrary at $950 \mathrm{~K}$ vacancy contribution is 
dominant and $\mathrm{Cr}$ depletion occur [9]. We will compare these trends with experimental observations of RIS. Finally, the AKMC enables to study the interaction between segregation and precipitation [9]: typical cases will be presented.

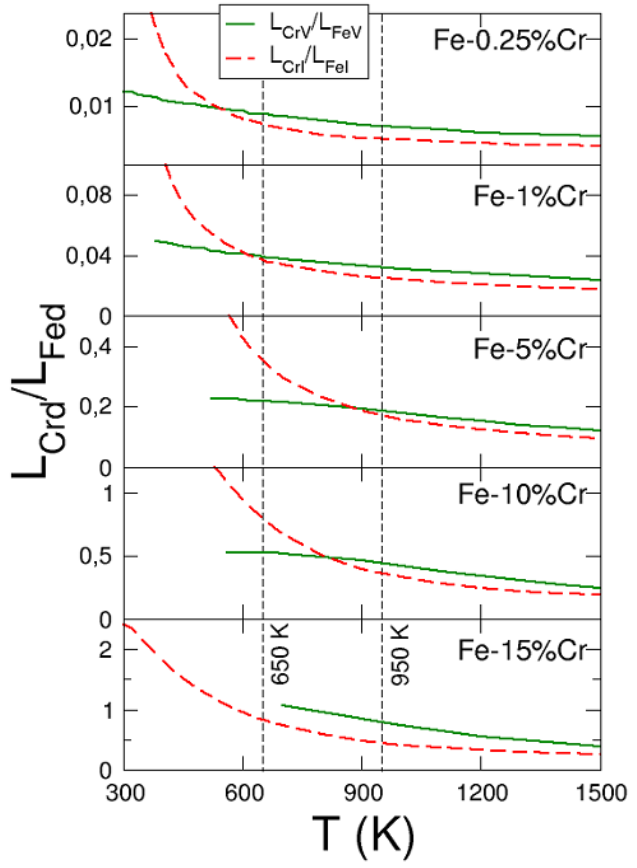

(a)

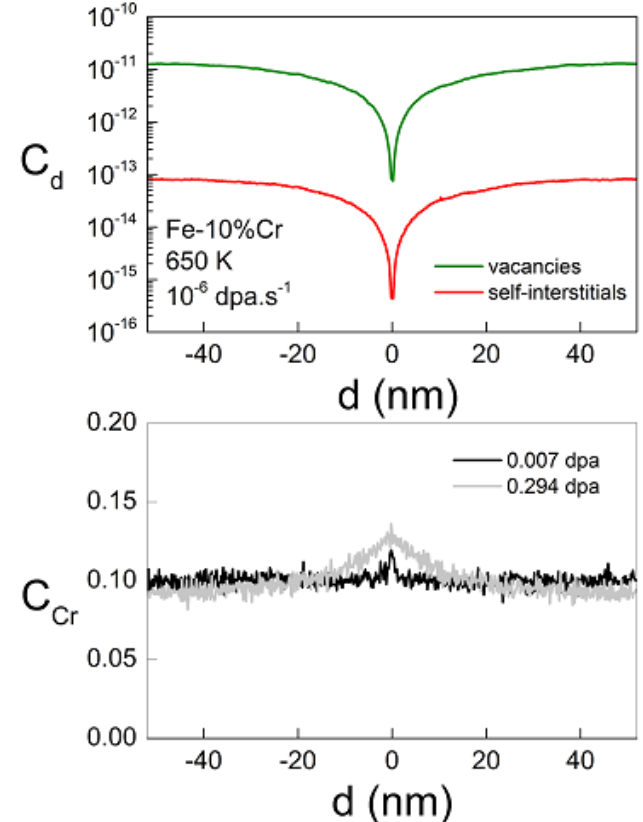

(b)

Fig. 1: (a) Evolution of the ratios of Onsager coefficients with the temperature, in Fe-Cr alloys of different compositions, estimated by Monte Carlo simulations, (b) Monte Carlo simulation of radiation induced segregation of $\mathrm{Cr}$ near a grain boundary (steady-state concentration profiles of point defects and concentration profiles of $\mathrm{Cr}$ at two successive doses).

\section{References}

[1] R.L. Klueh, A.T. Nelson, Ferritic/martensitic steels for next-generation reactors, J. Nucl. Mater. 371 (2007) 37.

[2] J.-L. Boutard et al, Oxide dispersion strengthened ferritic steels: a basic research joint program in France, J. Nucl. Mater. 455 (2014) 605.

[3] Z. Lu et al, Irradiation-induced grain boundary chromium microchemistry in high alloy ferritic steels, Scripta Mater. 58 (2008) 878

[4] M. Levesque, E. Martínez, C.-C. Fu, M. Nastar and F. Soisson, Simple concentration-dependent pair interaction model for large-scale simulations of $\mathrm{Fe}-\mathrm{Cr}$ alloys, Phys. Rev. B 84, 184205 (2011)

[5] O. Senninger, E. Martinez, F. Soisson, M. Nastar and Y. Bréchet, Atomistic simulations of decomposition kinetics in Fe-Cr alloys: influence of the magnetism, Acta Mater. 73, 97-106 (2014).

[6] E. Meslin et al, Cluster-dynamics modelling of defects in $\alpha$-iron under cascade damage conditions, J. Nucl. Mater. 382 (2008) 190.

[7] M. Bachhav, G. R. Odette, and E. A. Marquis, $\alpha^{\prime}$ precipitation in neutron-irradiated $\mathrm{Fe}-\mathrm{Cr}$ alloys, Scripta Mater. 74 (2014) 48.

[8] V. Kuksenko, C. Pareige and P. Pareige, Cr precipitation in neutron irradiated industrial purity Fe-Cr model alloys, J. Nucl. Mater. 432 (2013) 160.

[9] O. Senninger et. al., Modeling radiation induced segregation in $\mathrm{Fe}-\mathrm{Cr}$ alloys, submitted to Acta Mater. 
DE LA RECHERCHE À L'INDUSTRIE
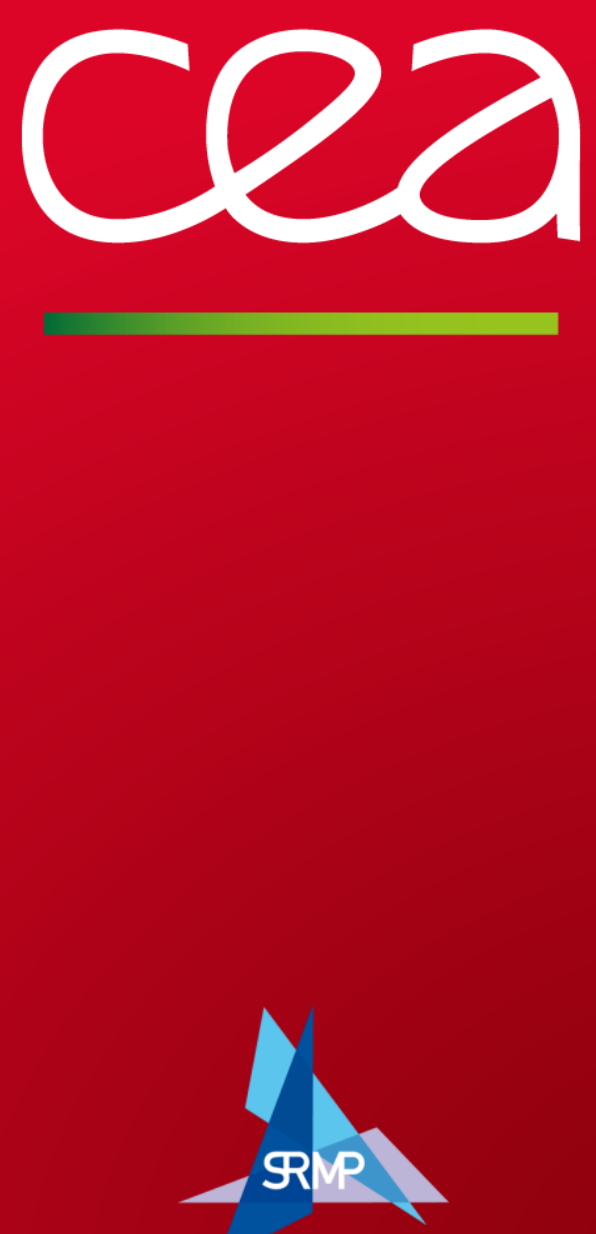

www.cea.fr

\section{Atomistic Modeling of Segregation and Precipitation in Fe-Cr Alloys under Irradiation}

C.-C. Fu, T. Jourdan, M. Nastar, O. Senninger, F. Soisson

SRMP, CEA Saclay

E. Martinez

Los Alamos National Laboratory

Y. Bréchet

SIMAP, Grenoble INP November 4-6, 2015, CEA - INSTN Cadarache, France 


\section{Fe-Cr alloys: nuclear applications}

\section{MINDS}

- Fe-Cr alloys : a model for ferritic and ferritic-martensitic steels $(7-18 \% \mathrm{Cr})$ candidate materials for future nuclear reactors (Gen IV and fusion)

Potential problems under irradiation:

- $\alpha$ precipitation $\rightarrow$ hardening and embrittlement

- $\mathrm{Cr}$ depletion at GBs $\rightarrow$ corrosion, embrittlement

Due to: point defect supersaturation

Acceleration of precipitation

Radiation Induced Segregation (RIS)

Radiation Induced Precipitation (RIP)

- Main objectives:

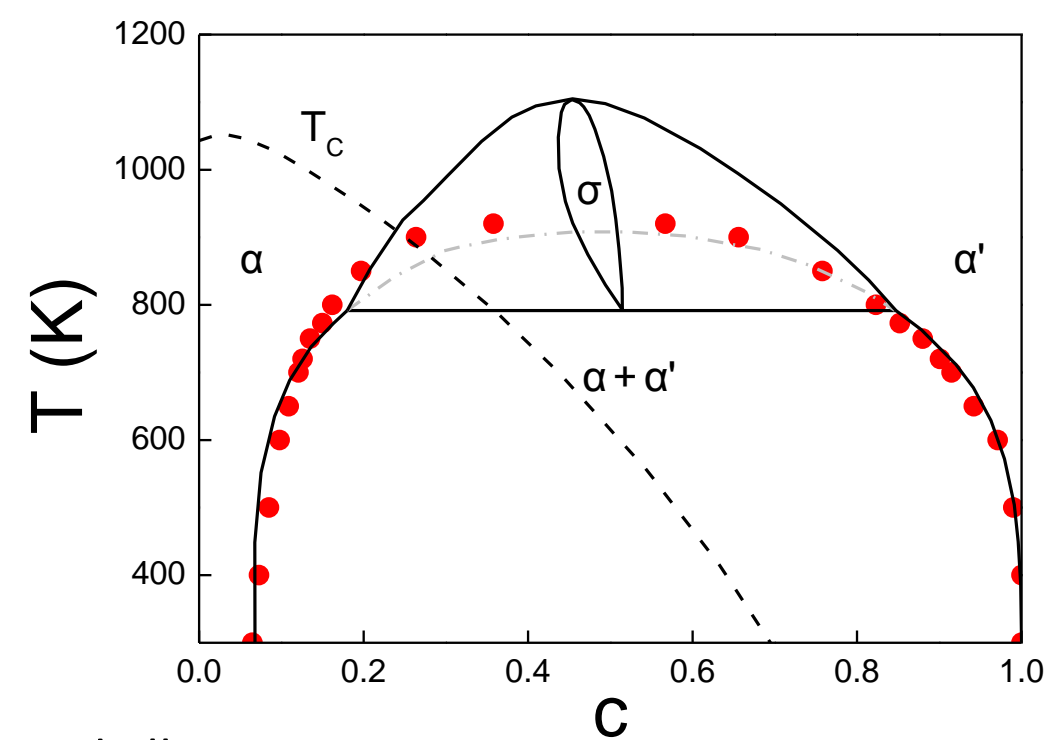

- quantify the acceleration of precipitation in supersaturated alloys

- understand the mechanisms controlling RIS

- study possible effects of equilibrium vs non-equilibrium segregation, ballistic mixing, carbon atoms,... on the precipitation and segregation kinetics

- Our approach : Atomistic Kinetic Monte Carlo (AKMC) simulations 


\section{Diffusion model - AKMC Simulations}

\section{MINDS}

Pair interactions on stable (bcc) and saddle-point positions

- Diffusion by jumps of :

- Vacancies (V)

- Self-interstitial atoms (SIA) : $<110>$ dumbbells

- Direct interstitials (C)

- The migration barriers are computed with a brokenbond model:

- with composition and temperature dependent pair interactions

- with saddle-point pair interactions

- fitted on DFT calculations

- Formation of isolated Frenkel with replacement collision sequences or small replacement cascades

- Annihilation of $\mathrm{V}$ and SIA on a perfect sink

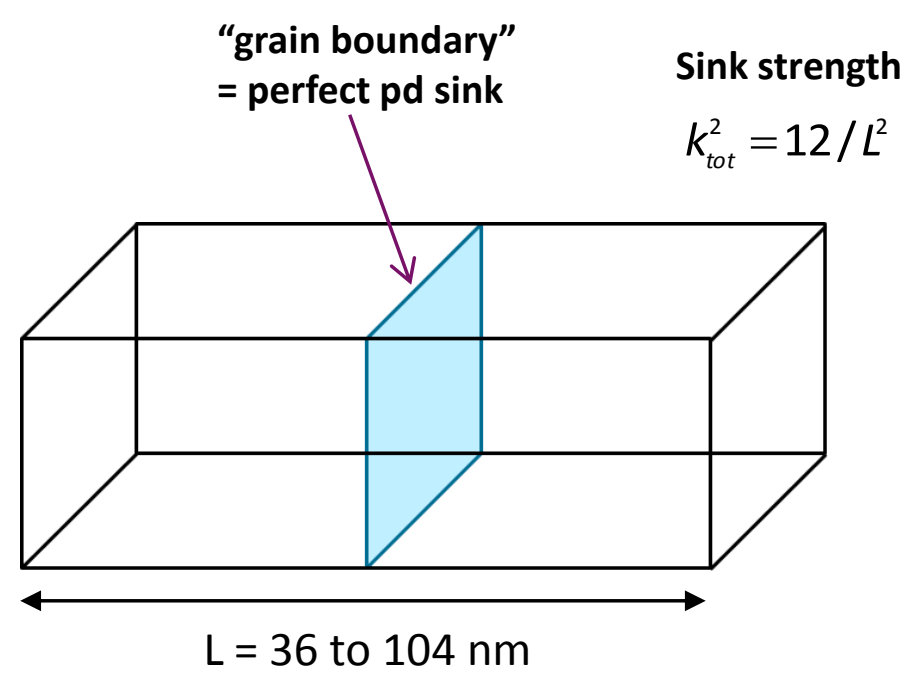




\section{Thermodynamics: effective pair interactions}

\section{$\because$ MINDS}

Concentration dependent pair interactions: $h_{F e F e}^{(n)}, h_{c r c r}^{(n)}, h_{F e c r}^{(n)}(x)$ fitted on DFT calculations of $\Delta \mathrm{H}_{\text {mix }}$ at $0 \mathrm{~K}$ Magnetic and vibrational contributions: linear temperature dependence, fitted on $\mathrm{T}_{\alpha-\alpha^{\prime}}$ (exp)

M. Levesque et al, Phys. Rev. B 84, 184205 (2011)

$$
\begin{aligned}
& g_{F e C r}^{(n)}(x, T)=h_{F e C r}^{(n)}(x)-T s_{F e c r}^{(n)}(x), \text { with } n=1,2 \\
& \Delta H_{\text {mix }}=\Omega(x) x(1-x) \\
& \Omega(x)=\sum_{n} \frac{z_{n}}{2}\left[h_{F e F e}^{(n)}+h_{C r C r}^{(n)}-2 h_{F e C r}^{(n)}(x)\right]
\end{aligned}
$$

\section{PWSCF, GGA-PAW}

- Special Quasi-random structures (SQS)

$\diamond$ Ordered structures

- Pair interaction model

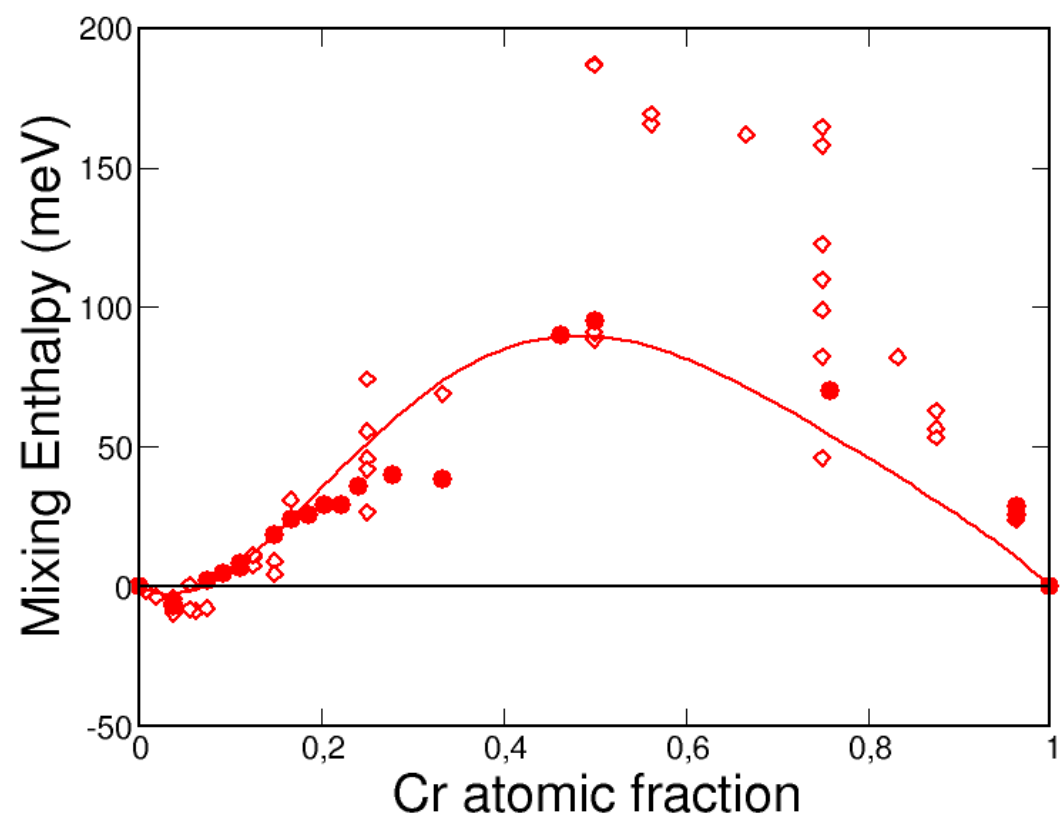

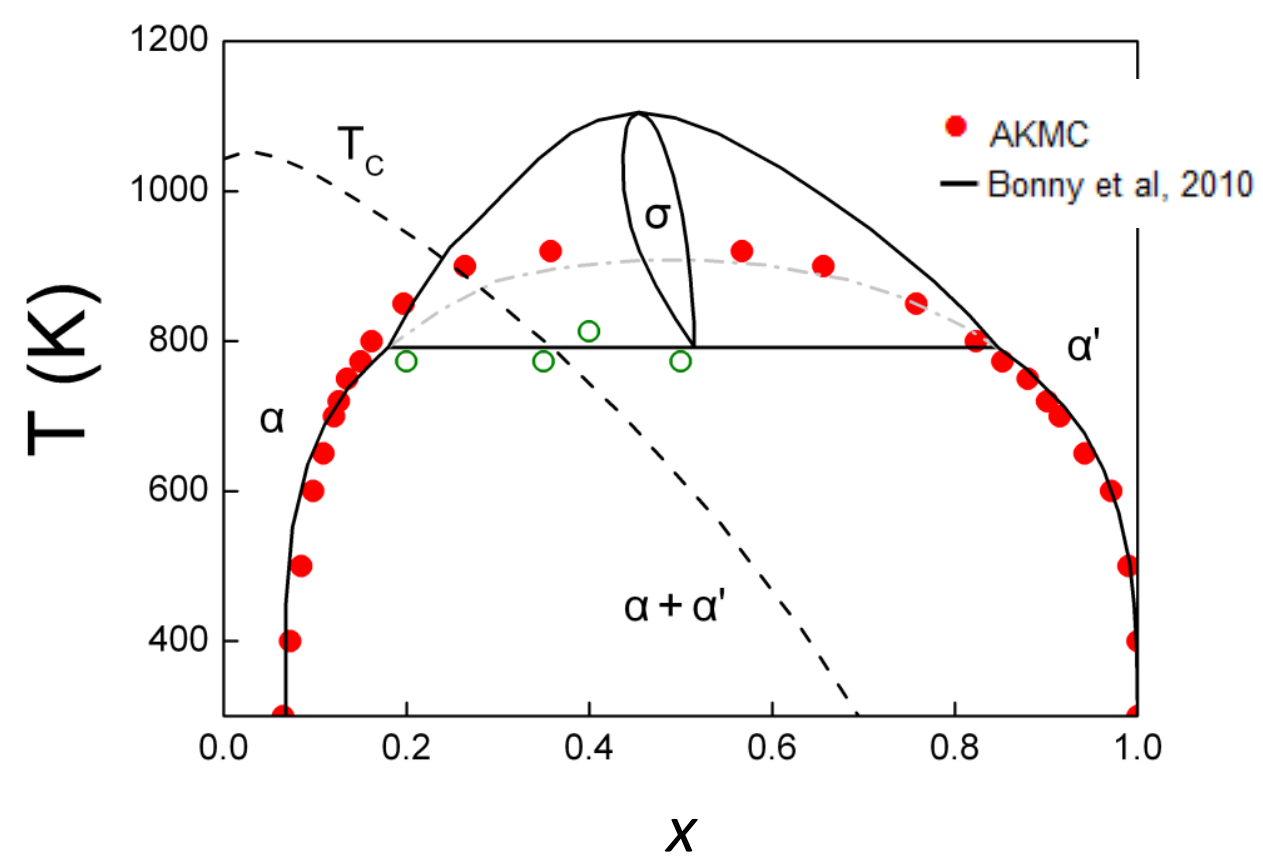




\section{CeaKinetics of $\alpha-\alpha$ ' decomposition: isothermal annealing}

\section{MIN口S}

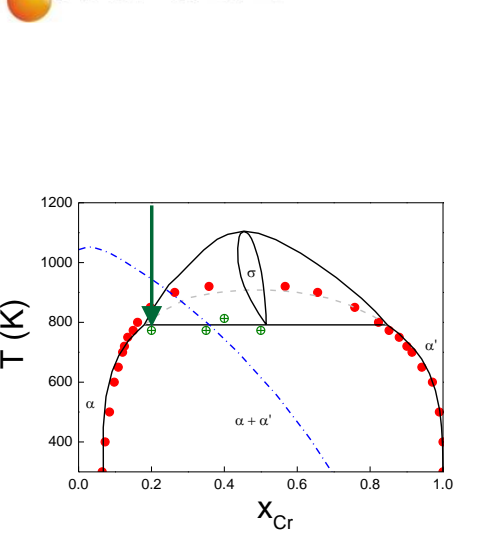

$\mathrm{Fe}-20 \% \mathrm{Cr} \quad \mathrm{T}=500^{\circ} \mathrm{C}$ AKMC (E. Martinez et al, 2012)

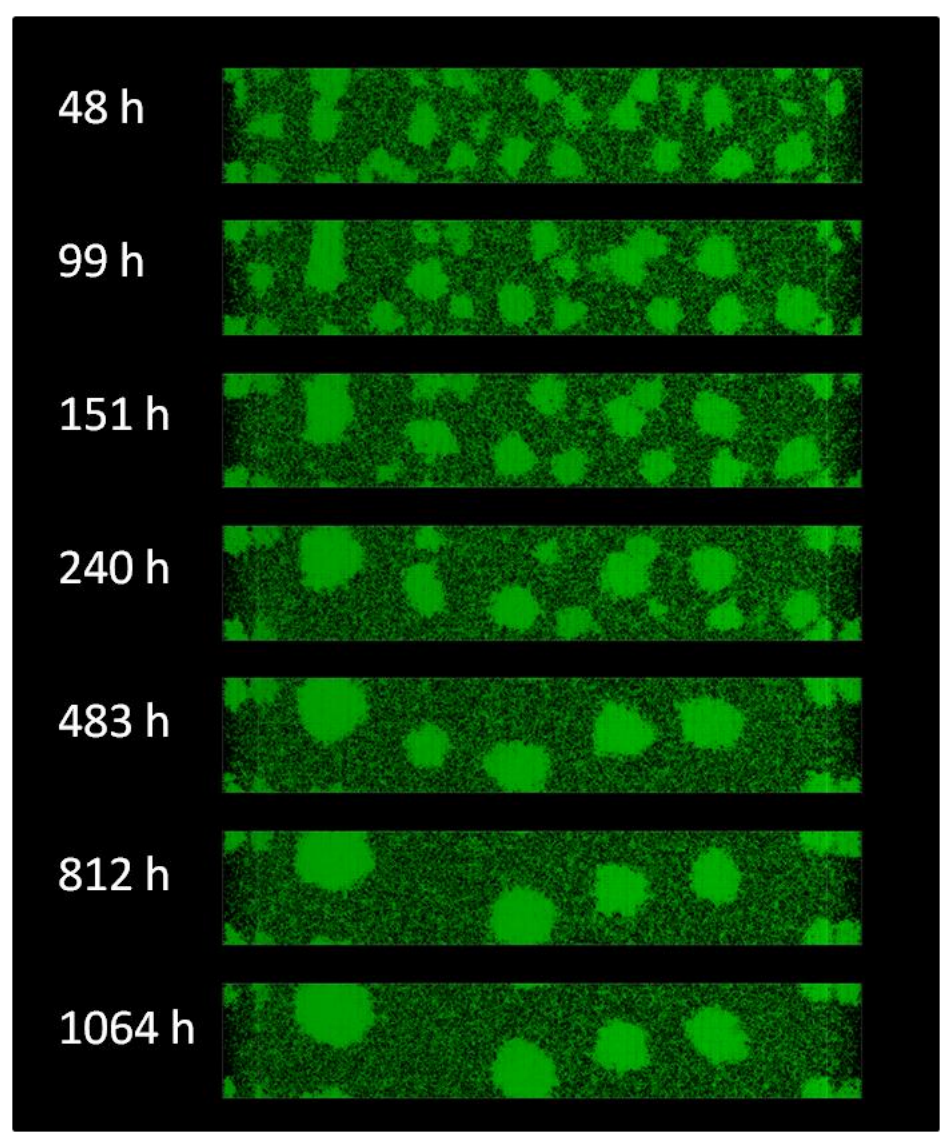

3DAP (Novy et al, 2009)

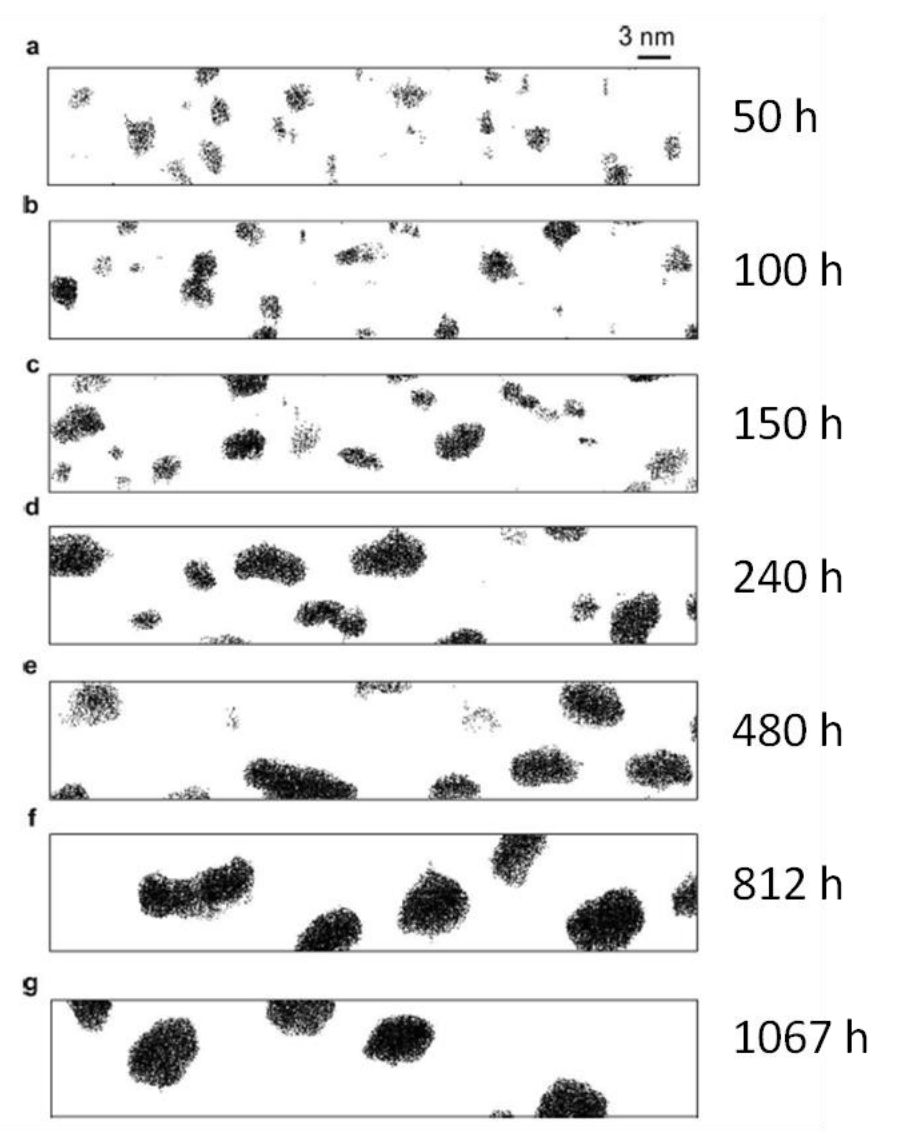

E. Martinez et al, Phys. Rev. B 86, 224109 (2012)

S. Novy et al, J. Nucl. Mater. 384 (2009) 96-102 


\section{Cea Kinetics of $\alpha-\alpha^{\prime}$ decomposition: isothermal annealing}

\section{$\because$ MINDS}

Small-angle neutron scattering experiments (SANS) $500^{\circ} \mathrm{C}$ : Bley (1992)

$540^{\circ} \mathrm{C}$ : Furusaka et al. (1986)
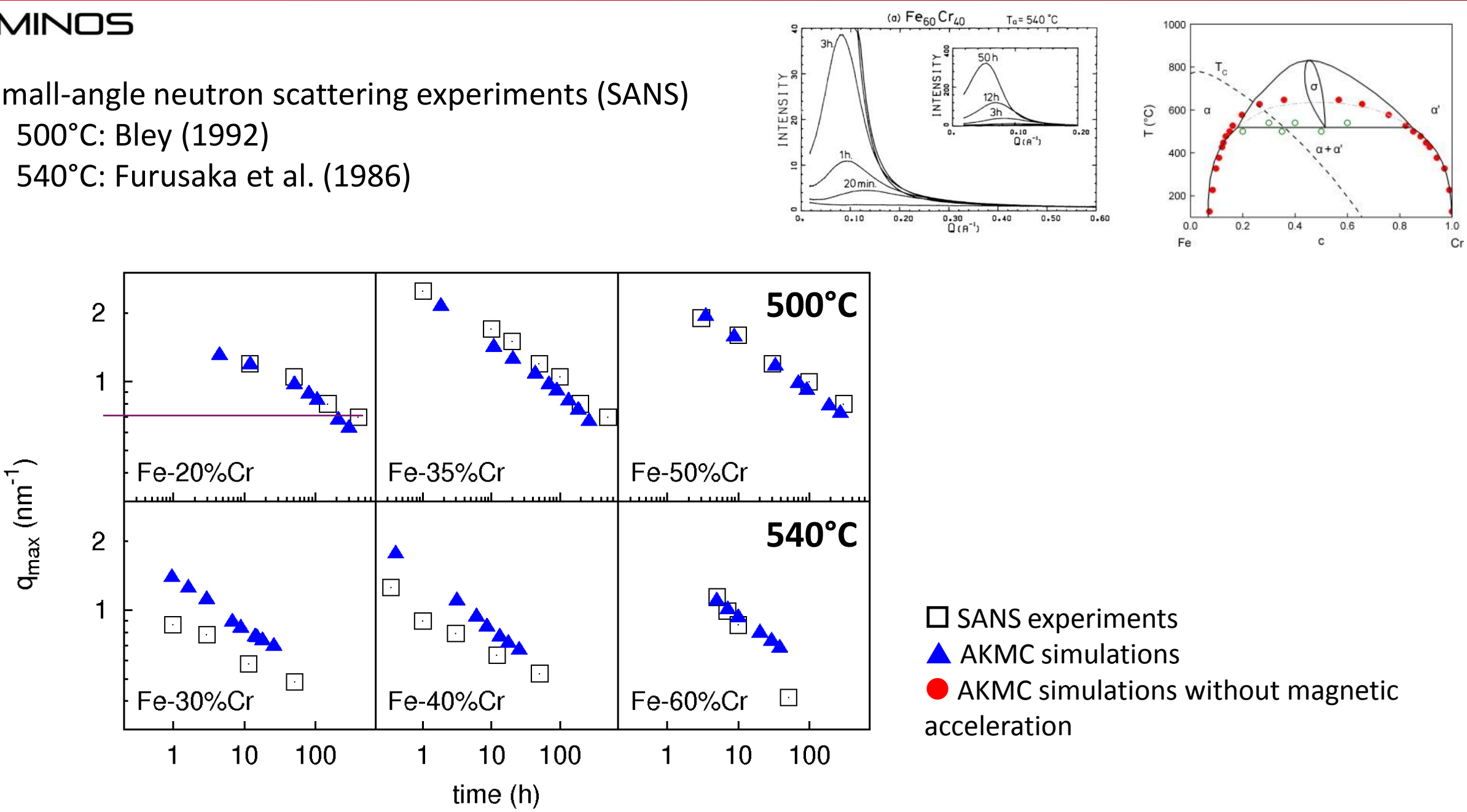

$\square$ SANS experiments

$\triangle \mathrm{AKMC}$ simulations

- AKMC simulations without magnetic acceleration

O. Senninger et al, Acta Mater. 73, 97-106 (2014) 


\section{Radiation Accelerated Precipitation: experiments}

\section{MINDS}

\section{Experimental evidences (3DAP)}

in Fe- $9 \%$ to $20 \% \mathrm{Cr}$ alloys at $290-300^{\circ} \mathrm{C}$

- isothermal annealing: $\alpha^{\prime}$ precipitation is not observed due to slow kinetics

- neutron irradiation: $\alpha^{\prime}$ precipitation

Bachhav et al (Scripta Mater 2014)

$\mathrm{Fe}-3 \% \mathrm{Cr}$ to $18 \% \mathrm{Cr}, \mathrm{T}=290^{\circ} \mathrm{C}$

$3.4 \times 10^{-7} \mathrm{dpa} / \mathrm{s}, 1.82 \mathrm{dpa}$

V. Kuksenko et al, JNM 432 (2013) 160

C. Pareige et al JNM 456 (2015) 471-476

Precipitation under neutron irradiation in $\mathrm{Fe}-9 \% \mathrm{Cr}$ and $\mathrm{Fe}-12 \% \mathrm{Cr}$, but not under ion irradiation at higher flux (a)

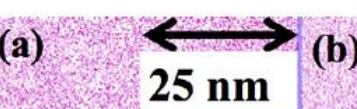

(b)

(c)
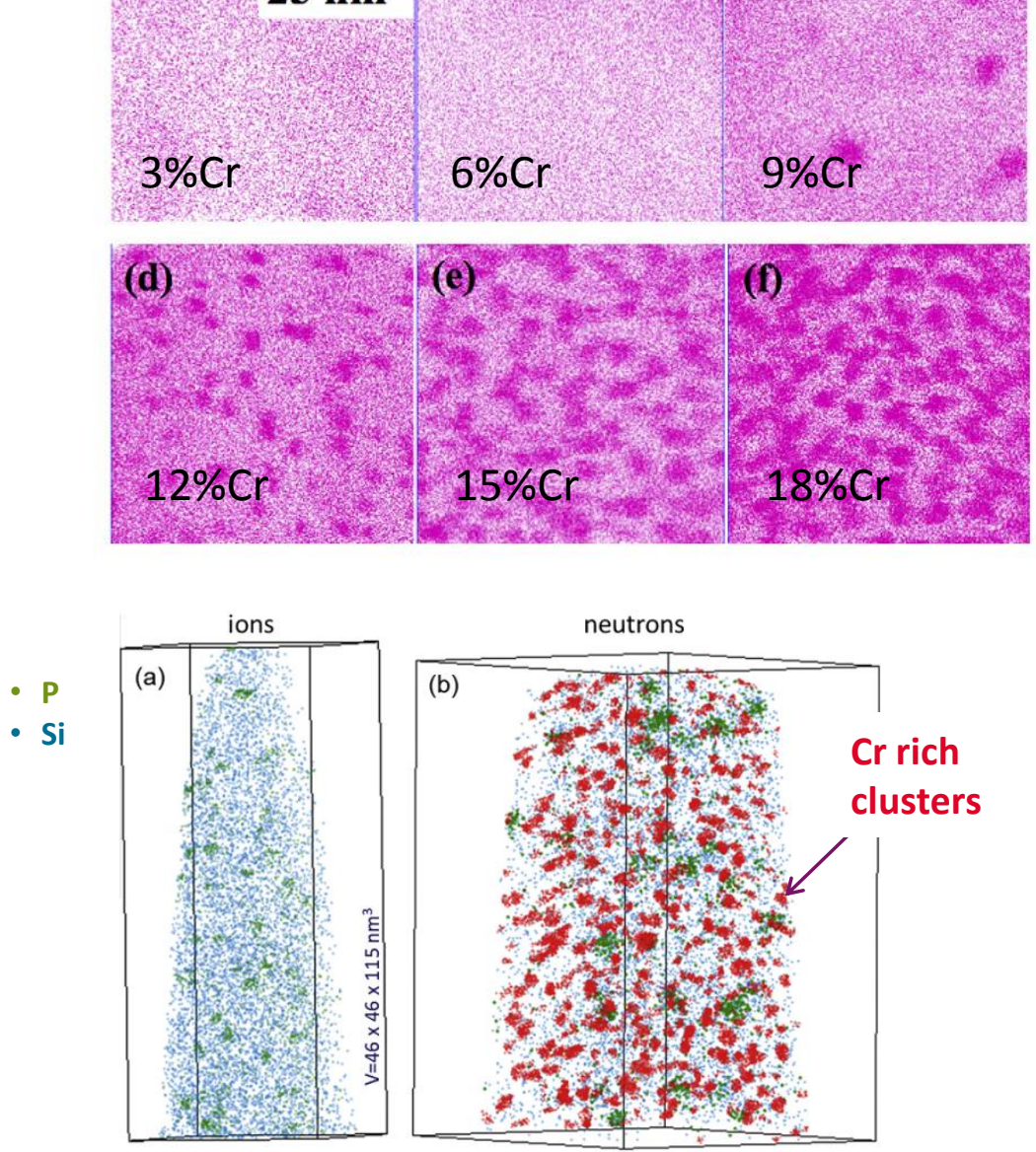

$300^{\circ} \mathrm{C}-0.5 \mathrm{dpa}$

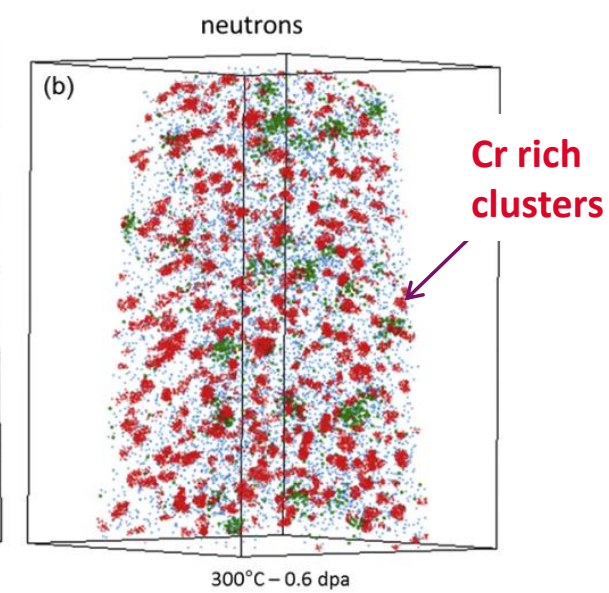




\section{Radiation Accelerated Precipitation: AKMC}

\section{MINDS}

AKMC simulations

Fe-18\%Cr@ 563 K, 3.4 x 10-7 dpa.s ${ }^{-1}$

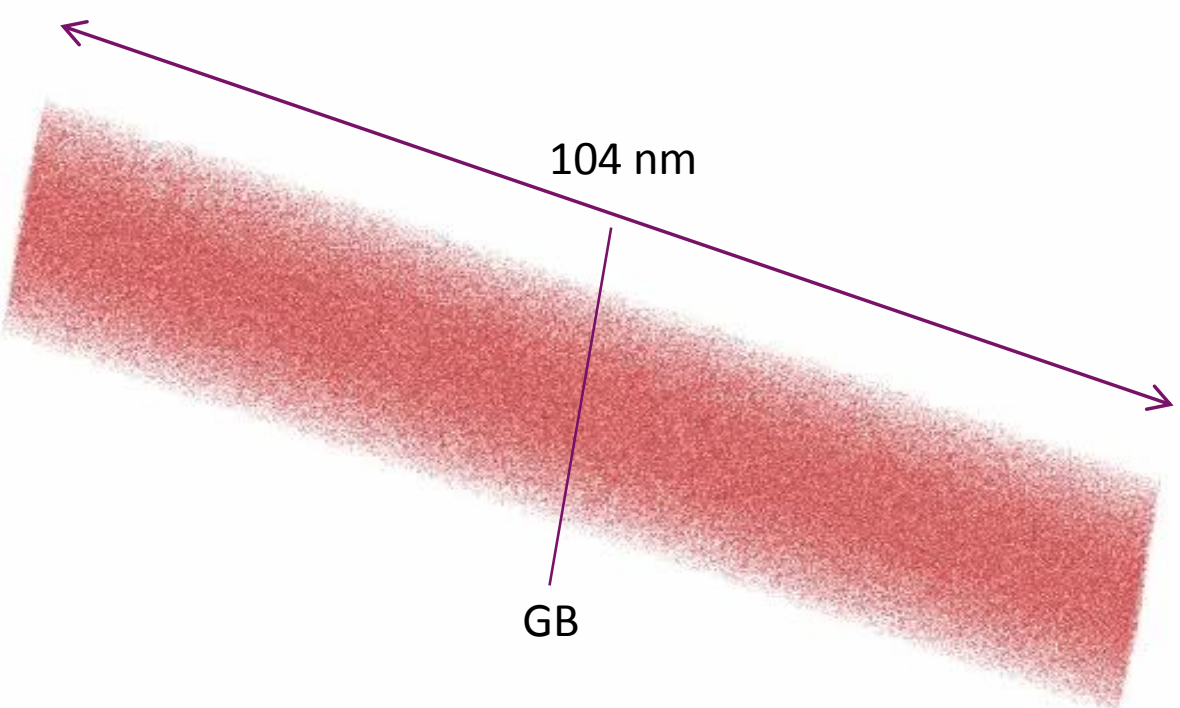

Precipitate free zones near the GBs
Point defect concentration profiles
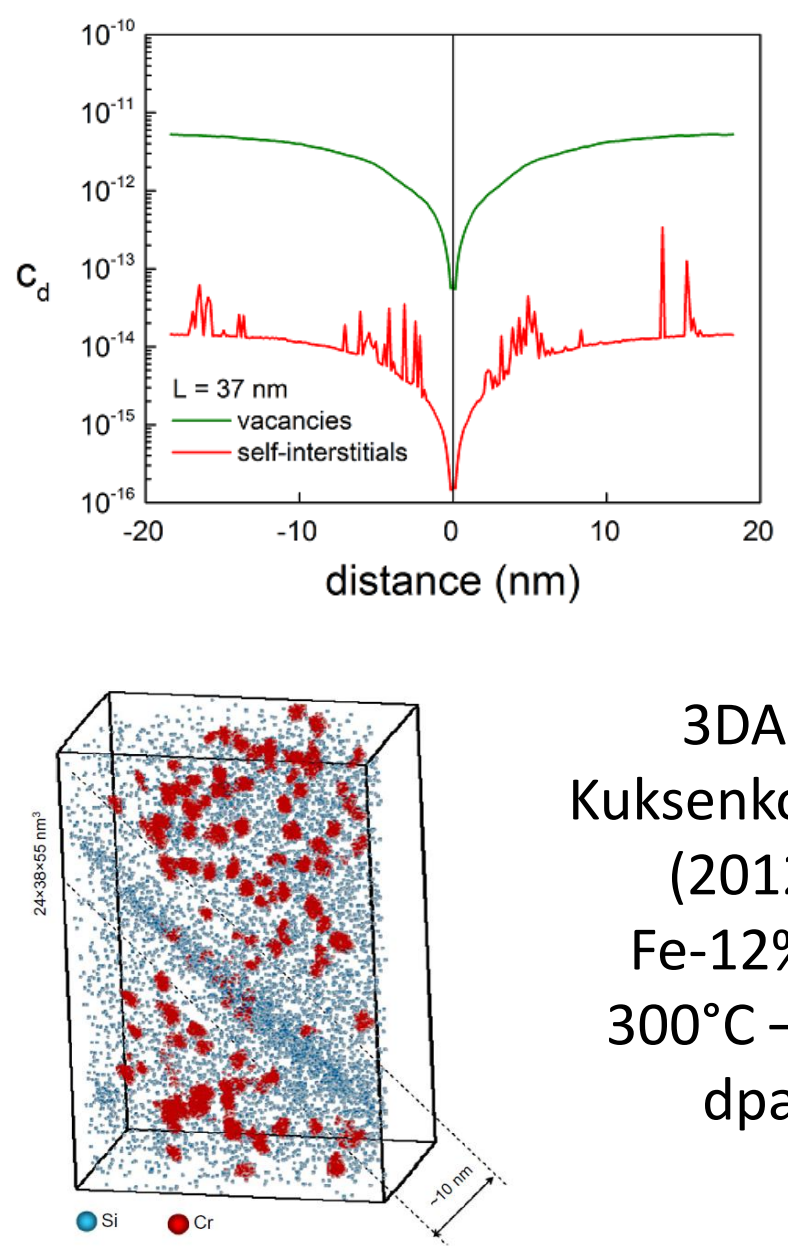

3DAP

Kuksenko et al

(2012)

$\mathrm{Fe}-12 \% \mathrm{Cr}$ $300^{\circ} \mathrm{C}-0.6$ dpa 


\section{Cea Radiation Accelerated Precipitation: AKMC vs 3DAP}

\section{MINDS}

- AKMC simulations: one GB in the simulation box (constant sink strength: $k_{t o t}^{2}=12 / L^{2}$ )

- Cluster Dynamics: evolution of the sink strength $k_{\text {tot }}^{2}$ (in pure iron)

$\rightarrow$ better estimation of the point defect concentration $c_{i, v}=G /\left(k_{t o t}^{2} D_{i, v}\right)$

$\rightarrow$ rescaling of the Monte Carlo time scale

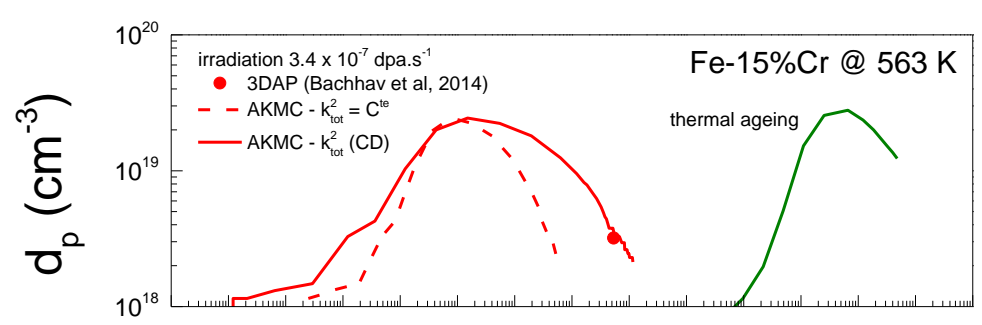

$$
t=t_{M C} \times \frac{c_{i, v}(A K M C)}{c_{i, v}(C D)}
$$
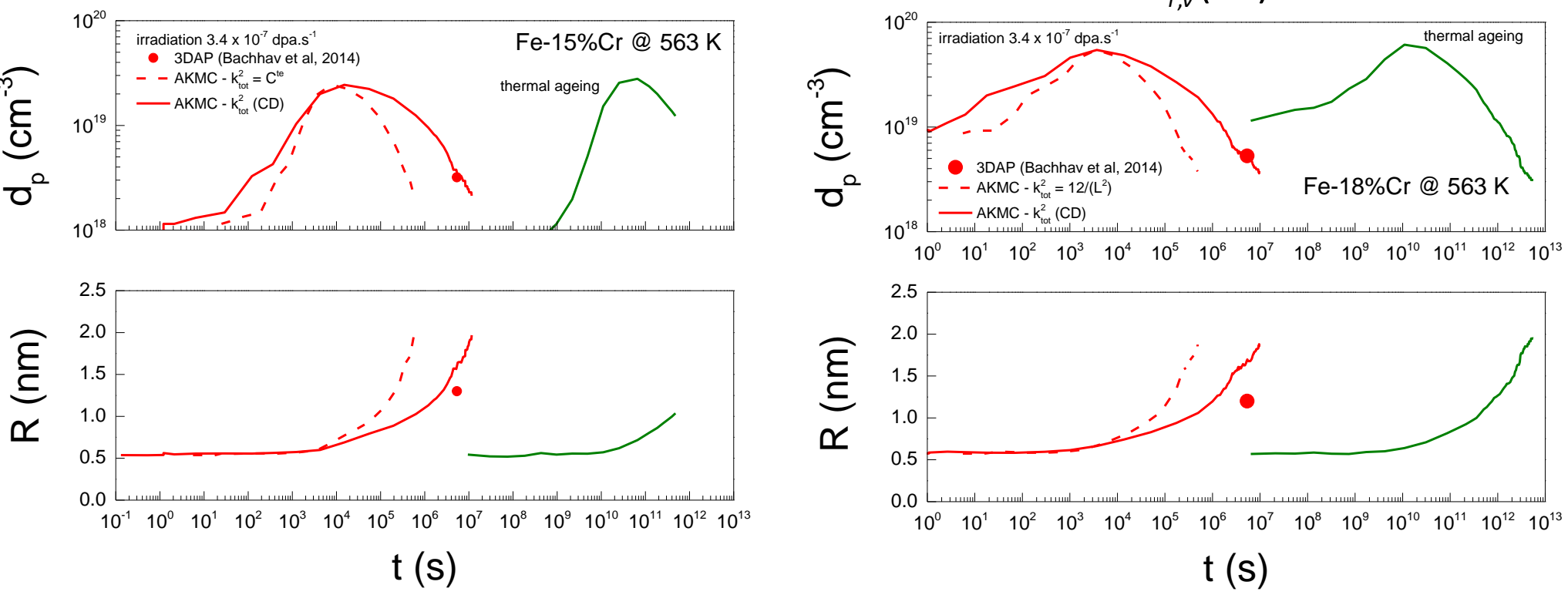

Strong acceleration by irradiation $\left(\times 10^{6}-10^{7}\right)$ Good agreement with the experiments of neutron irradiation (Bachhav et al, 2014)

F. Soisson, T. Jourdan, Acta Mater 2016. 


\section{Effect of ballistic mixing}

\section{MINDS}

- Experiments: $\mathrm{Cr}$ precipitation under neutron irradiation, but not under ion irradiation at higher flux A ballistic dissolution of $\alpha^{\prime}$ precipitates, in displacement cascades ?

- $A K M C$ simulations

- at different dose rates $G\left(\right.$ dpa. $\left.s^{-1}\right)$

- with different numbers of replacements/displacement

$\mathrm{N}_{\text {rep }} / \mathrm{N}_{\text {dis }}=0 \quad$ (channeling)

$\mathrm{N}_{\mathrm{rep}} / \mathrm{N}_{\mathrm{dis}}=10 \quad$ (replacement collision sequences)

$\mathrm{N}_{\text {rep }} / \mathrm{N}_{\text {dis }}=100$ (cascades)

- At $290^{\circ} \mathrm{C} \quad \gamma=\frac{\Gamma_{\text {bal }}}{\left\langle\Gamma_{\text {th }}\right\rangle} \propto \frac{G}{c_{v}^{s t} D_{v}} \square 10^{-3}-10^{-4}$

- no dissolution of precipitates

- no effect of $\mathrm{N}_{\text {rep }} / \mathrm{N}_{\text {dis }}$ on the precipitation kinetics - dose rate effects: a simple acceleration due to the point defect supersaturation

$$
c_{i, v}=G /\left(k_{t o t}^{2} D_{i, v}\right)
$$

$\rightarrow$ ballistic effects do not explain the difference between ion and neutron irradiations
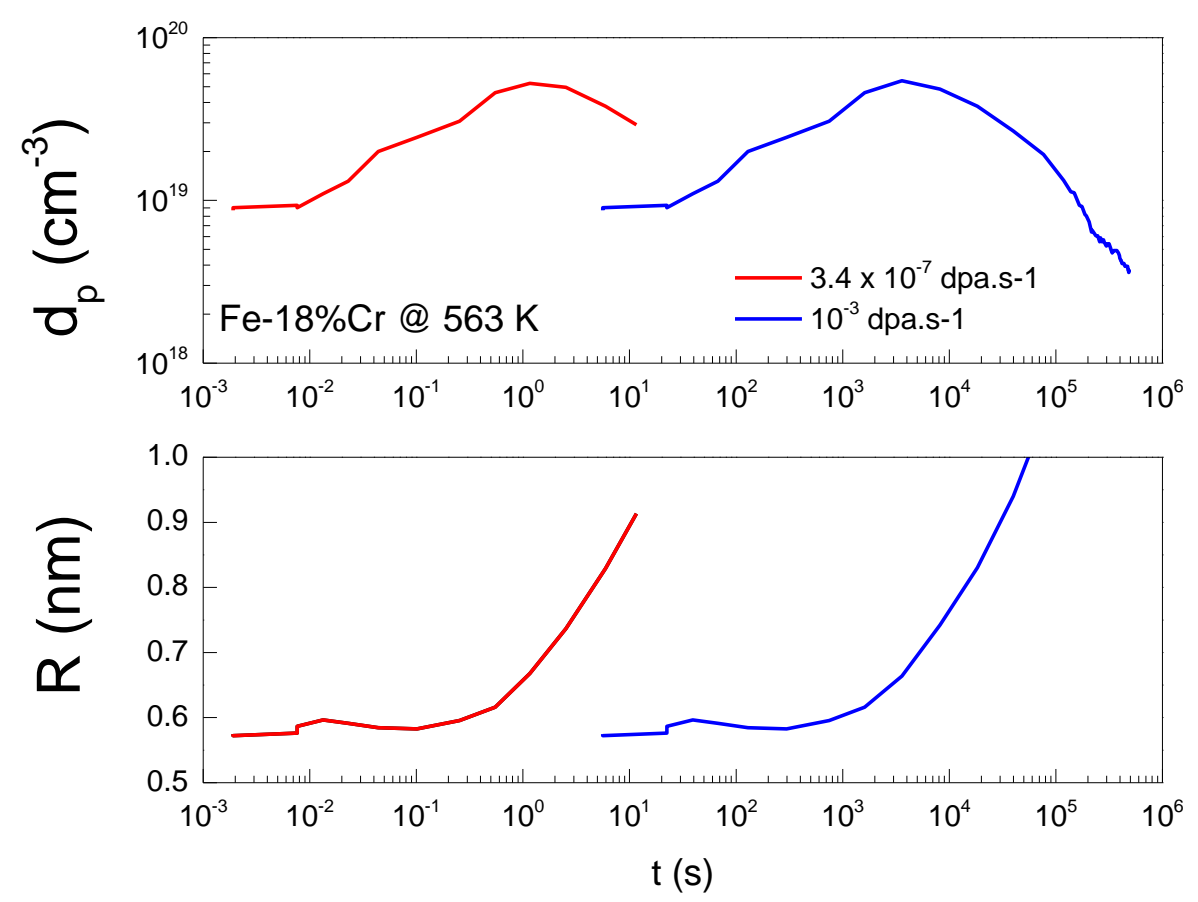

- Possible ballistic effects at lower temperatures (below $100^{\circ} \mathrm{C}$ ) 


\section{Radiation-Induced Segregation}

\section{$\because$ MINDS}

$$
J_{i}=-\sum_{j} L_{i j} \nabla \mu_{j}
$$

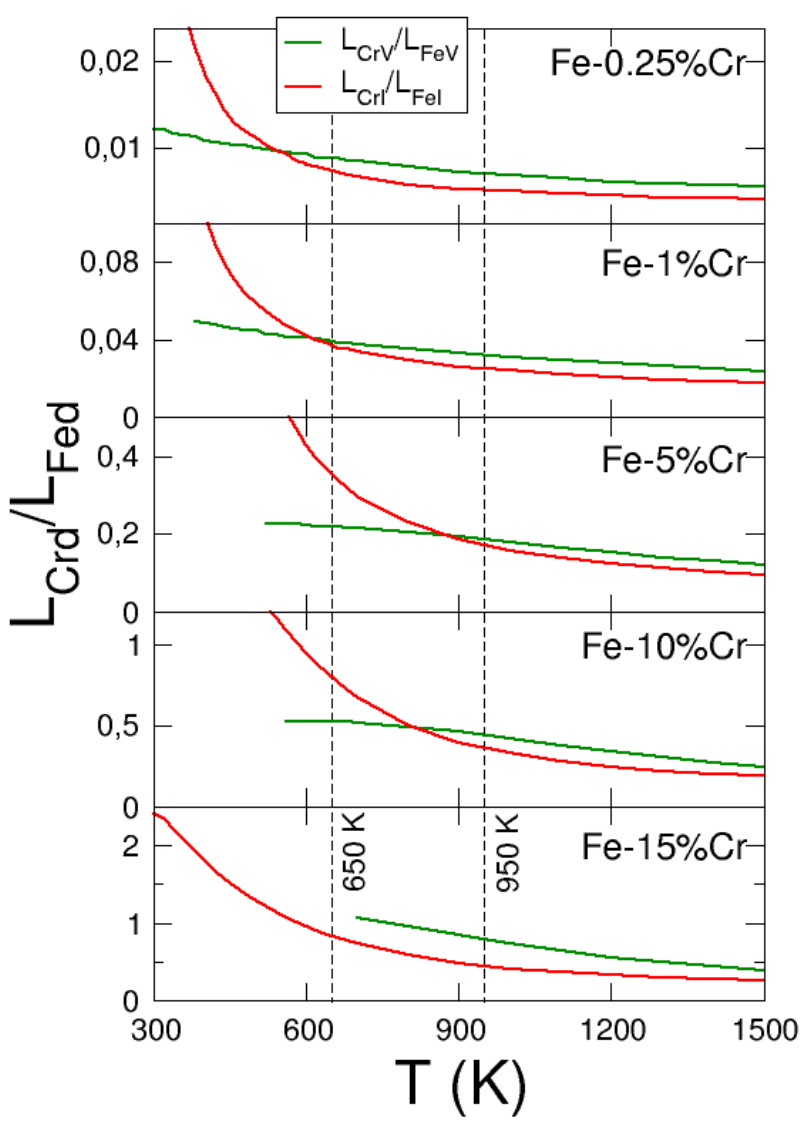

Steady-state profile:

$\nabla C_{C r} \propto-\left(\frac{L_{C r V}}{L_{F e V}}-\frac{L_{C r l}}{L_{F e l}}\right) \nabla C_{V}$
$\mathrm{J}_{\mathrm{V}} / \mathrm{J}_{\mathrm{Cr}}$ : negative coupling $\rightarrow \mathrm{Cr}$ depletion at sinks, dominant at high $\mathrm{T}$ $\mathrm{J}_{\mathrm{SIA}} / \mathrm{J}_{\mathrm{Cr}}$ : positive coupling $\rightarrow \mathrm{Cr}$ enrichment at sinks, dominant at low $\mathrm{T}$

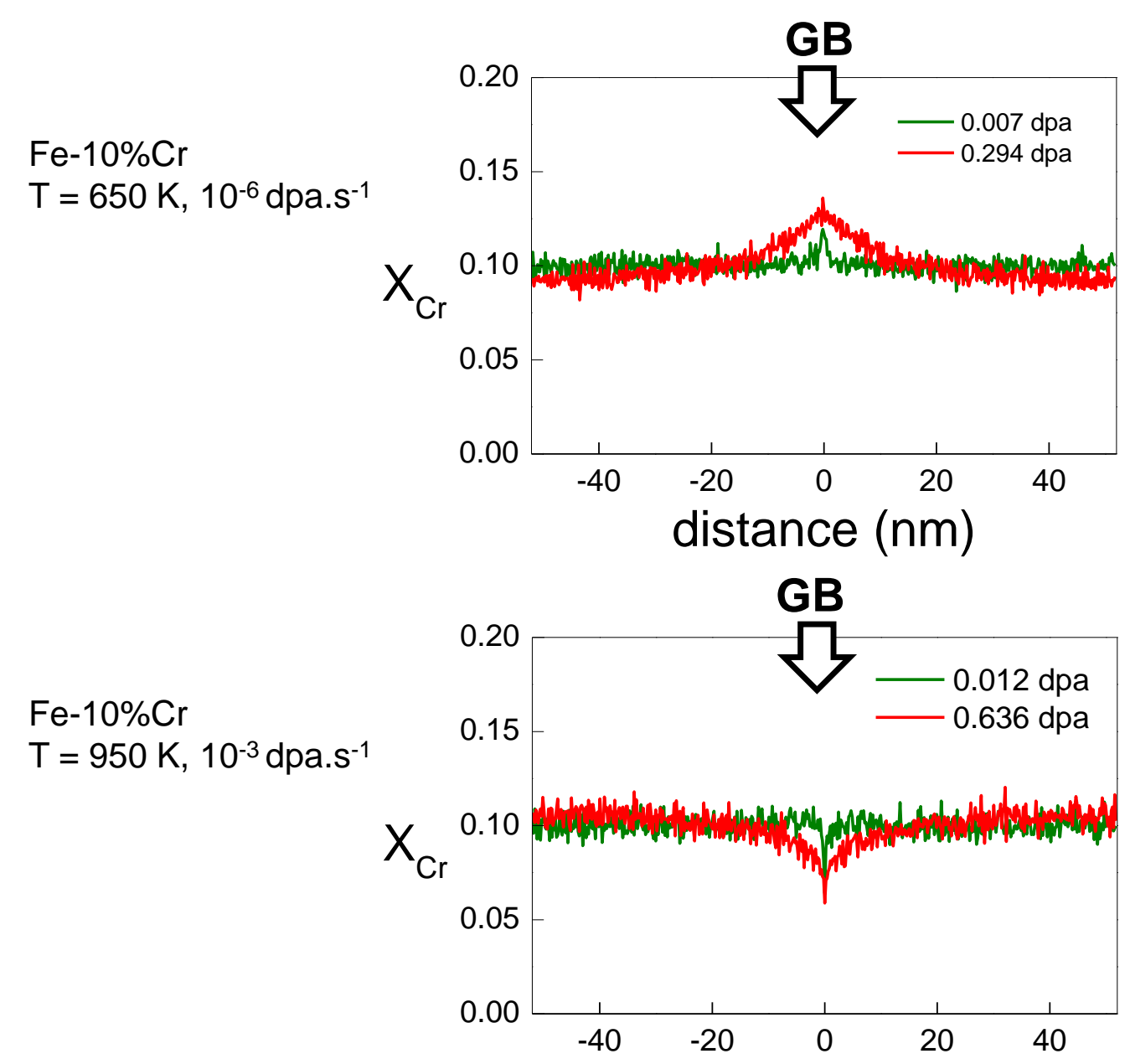

O. Senninger et al Acta Materialia 103, 1-11 (2016) 


\section{Radiation-Induced Segregation}

\section{$\because$ MINDS}

$$
J_{i}=-\sum_{\mathrm{j}} L_{i j} \nabla \mu_{\mathrm{j}}
$$

$\mathrm{J}_{\mathrm{V}} / \mathrm{J}_{\mathrm{Cr}}$ : negative coupling $\rightarrow \mathrm{Cr}$ depletion at sinks, dominant at high $\mathrm{T}$ $\mathrm{J}_{\mathrm{SIA}} / \mathrm{J}_{\mathrm{Cr}}$ : positive coupling $\rightarrow \mathrm{Cr}$ depletion at sinks, dominant at low $\mathrm{T}$

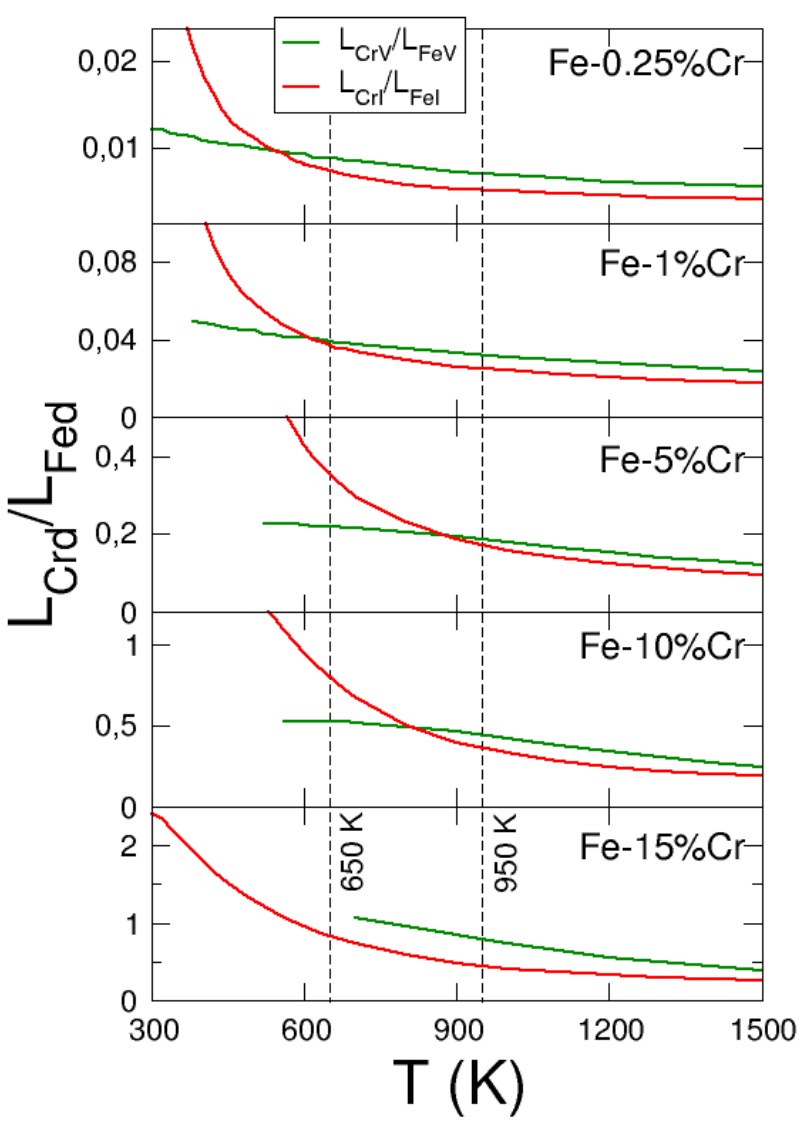

Steady-state profile:

$\nabla C_{C r} \propto-\left(\frac{L_{C r V}}{L_{F e V}}-\frac{L_{C r l}}{L_{F e l}}\right) \nabla C_{V}$

$$
e_{c r}^{s e g}=-0.1 \mathrm{eV}
$$

$\mathrm{Fe}-10 \% \mathrm{Cr}$

$\mathrm{T}=650 \mathrm{~K}, 10^{-6} \mathrm{dpa}_{\mathrm{s}} \mathrm{s}^{-1}$

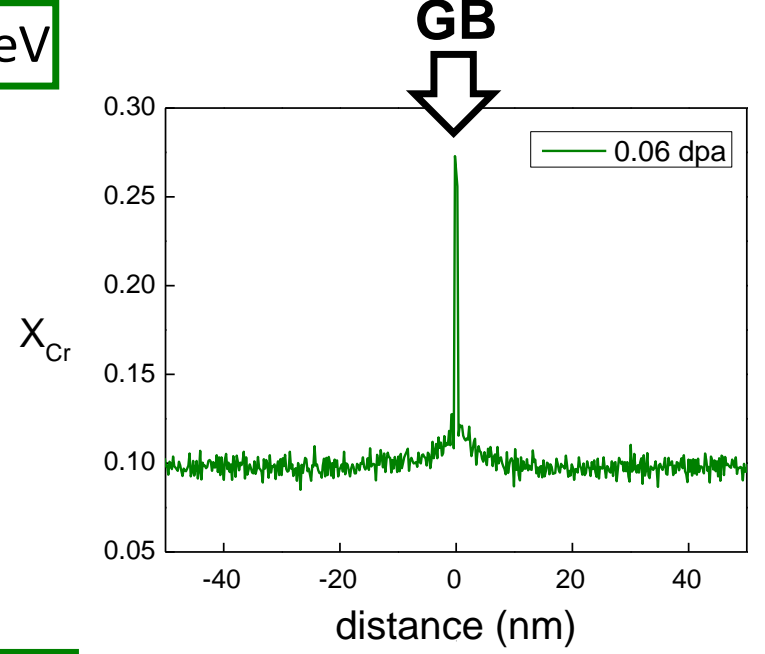

$e_{c r}^{s e g}=-0.1 \mathrm{eV}$

$\mathrm{Fe}-10 \% \mathrm{Cr}$

$\mathrm{T}=950 \mathrm{~K}, 10^{-3} \mathrm{dpa}_{\mathrm{s}} \mathrm{s}^{-1}$

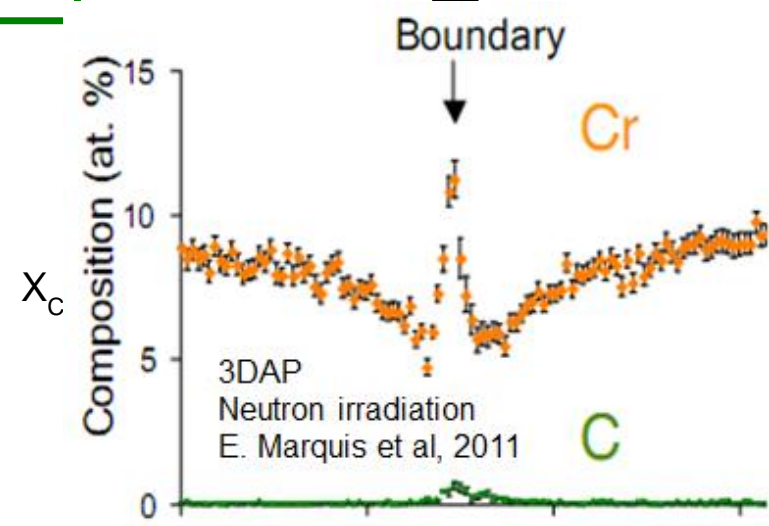

W-shape profile 


\section{Radiation-Induced Precipitation (AKMC)}

\section{MINDS}

Undersaturated alloys, at low $\mathrm{T}:$ strong $\mathrm{Cr}$ enrichment on sinks $\rightarrow$ RIS $\rightarrow$ radiation induced precipitation

$\mathrm{T}=563 \mathrm{~K}$ $\mathrm{Fe}-9 \% \mathrm{Cr}$ $10^{-6}$ dpa.s s $^{-1}$
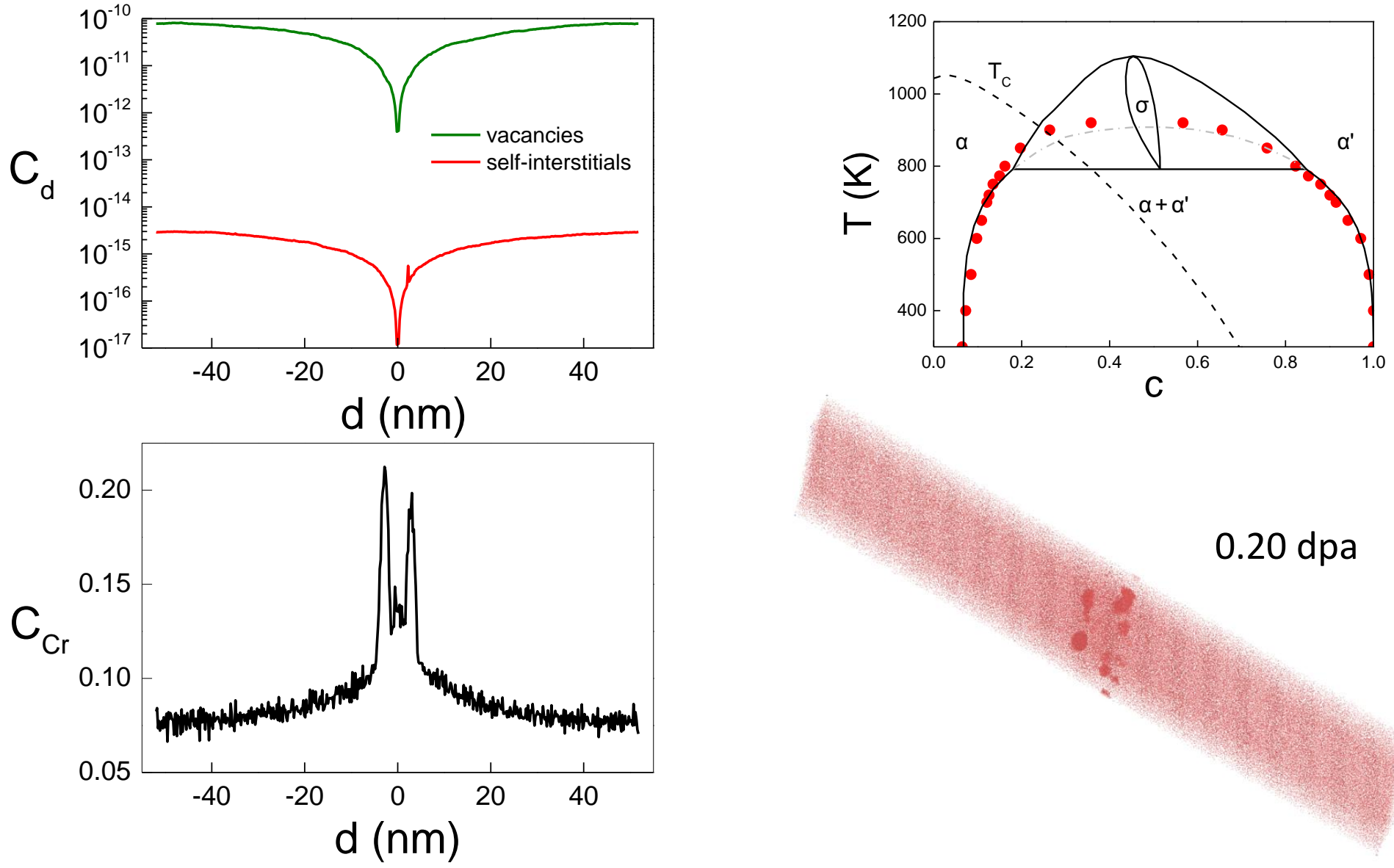

$0.20 \mathrm{dpa}$ 


\section{CONCLUSIONS}

\section{MINDS}

AKMC simulations with thermodynamic and point defect parameters fitted on DFT calculations Good description of driving forces, diffusion properties and nucleation

\section{In Fe-Cr alloys}

- Magnetic effects are important (impact on thermodynamic and diffusion properties)

- Radiation Induced Segregation is controlled by a balance between opposite effects of $V$ and SIA the $L_{i j}$ are very dependent of the details of migration barriers

$\rightarrow$ may explain the variability of experimental studies ( $\neq$ RIS in austenitic steels)

\section{- Radiation accelerated precipitation}

- a good agreement with neutron irradiations (with rescaling of the sink strength using CD)

- no effect ballistic mixing in the experimental conditions (relatively high temperatures, high sink densities) $\rightarrow$ does not explain the difference between neutron an on irradiation

- precipitation kinetics is less sensitive than Radiation Induced Segregation to the details of migration barriers

\section{- Related work, perspectives}

- Phase-Field model for RIS (J.B. Piochaud, L. Thunier, A. Legris - UMET, Lille)

- Precipitation under electron irradiation (O. Tissot, et al)

- Effects of $\mathrm{C}($ or $\mathrm{O}, \mathrm{N})$ on the kinetics of precipitation and segregation 